RNA WORLD

\section{Behind the scenes}

In the past decade there has been a surge of interest in microRNAs (miRNAs) - tiny, non-coding molecules of RNA. miRNAs have two functions: they base pair with partially complementary mRNAs to prevent the translation of $\mathrm{mRNA}$ into protein, and they reduce the cellular concentration of their mRNA targets. However, despite the significant insights into gene regulation that have been gleaned from the study of miRNAs, their modes of action have remained poorly understood. Two papers now highlight new biological functions and regulatory mechanisms of miRNAs.

Giraldez and colleagues set out to identify the in vivo targets of miRNAs, which are largely unknown. Using microarrays and in vivo target validation, the authors identified a large group of mRNAs that had a $>85 \%$ probability of being direct targets of the miRNA miR- 430 . They estimated that, during early zebrafish development, there are, in fact, several hundred direct targets of miR-430 regulation. An analysis of the target set showed that most were expressed maternally, which indicated that miR- 430 could have a crucial role in the maternal-tozygotic transition in embryogenesis. Poly(A) tails stabilize mRNAs and enhance translation, and deadenylation can trigger translational silencing and mRNA decay. Giraldez et al. tested whether the decay of miRNA targets correlated with changes in the poly(A) tail length of mRNAs. They showed that miR-430 does indeed accelerate the deadenylation of target mRNAs. Moreover, the group showed that impaired translation is not sufficient on its own to signifi- cantly increase deadenylation, nor is translation required for $\mathrm{miR}-430$ to increase the rate of poly $(\mathrm{A})$ tail removal.

In the second paper, Wu et al. examined two different miRNAs, miR-125b and let-7, in mammalian cells, and found that both miRNAs accelerate the decay of mRNA by a mechanism that is distinct from that of small interfering RNAs, which target mRNAs that are perfectly complementary. They showed that the miRNAs interact with the $3^{\prime}$ untranslated region of target mRNA and, similar to miR-430 in the Science study, induced accelerated mRNA degradation by expediting poly(A) tail removal. Interestingly, these miRNAs can induce this process for many mRNAs that bear various elements to which the miRNAs are partially complementary. Wu et al. also showed that the reduced translation and increased deadenylation are distinct. These findings indicate that miRNAs downregulate gene expression by two independent mechanisms: reduced translation efficiency and accelerated mRNA deadenylation and decay.

These two papers have identified possible mechanisms for miRNA function and have provided a fascinating behind-the-scenes look at the workings of miRNAs, which should continue to stoke the fire of interest in this 'tiny RNA world'.

Sharon Ahmad, Assistant Editor, Nature Reviews Molecular Cell Biology

ORIGINAL RESEARCH PAPERS Giraldez A. J. et al. Zebrafish miR-430 promotes deadenylation and clearance of maternal mRNAs. Science 16 February 2006 (doi:10.1126/science.1122689)| Wu, L. et al. MicroRNAs direct rapid

deadenylation of mRNA. Proc. Natl Acad. Sci. USA 22 February 2006 (doi:10.1073/pnas.0510928103) 